\title{
Independent tuning of excitonic emission energy and decay time in single semiconductor quantum dots
}

B. Höfer, J. Zhang, J. Wildmann, E. Zallo, R. Trotta, F. Ding, A. Rastelli, and O. G. Schmidt

Citation: Appl. Phys. Lett. 110, 151102 (2017);

View online: https://doi.org/10.1063/1.4979481

View Table of Contents: http://aip.scitation.org/toc/apl/110/15

Published by the American Institute of Physics

\section{Articles you may be interested in}

Quantum interference of two photons emitted from a luminescence center in GaAs: N

Applied Physics Letters 110, 152102 (2017); 10.1063/1.4979520

Electrically driven and electrically tunable quantum light sources

Applied Physics Letters 110, 071102 (2017); 10.1063/1.4976197

Single photon extraction from self-assembled quantum dots via stable fiber array coupling Applied Physics Letters 110, 142104 (2017); 10.1063/1.4979827

Two-photon interference from remote deterministic quantum dot microlenses

Applied Physics Letters 110, 011104 (2017); 10.1063/1.4973504

Optical probing of the Coulomb interactions of an electrically pumped polariton condensate Applied Physics Letters 110, 151103 (2017); 10.1063/1.4979836

Three-terminal quantum-dot thermal management devices

Applied Physics Letters 110, 153501 (2017); 10.1063/1.4979977

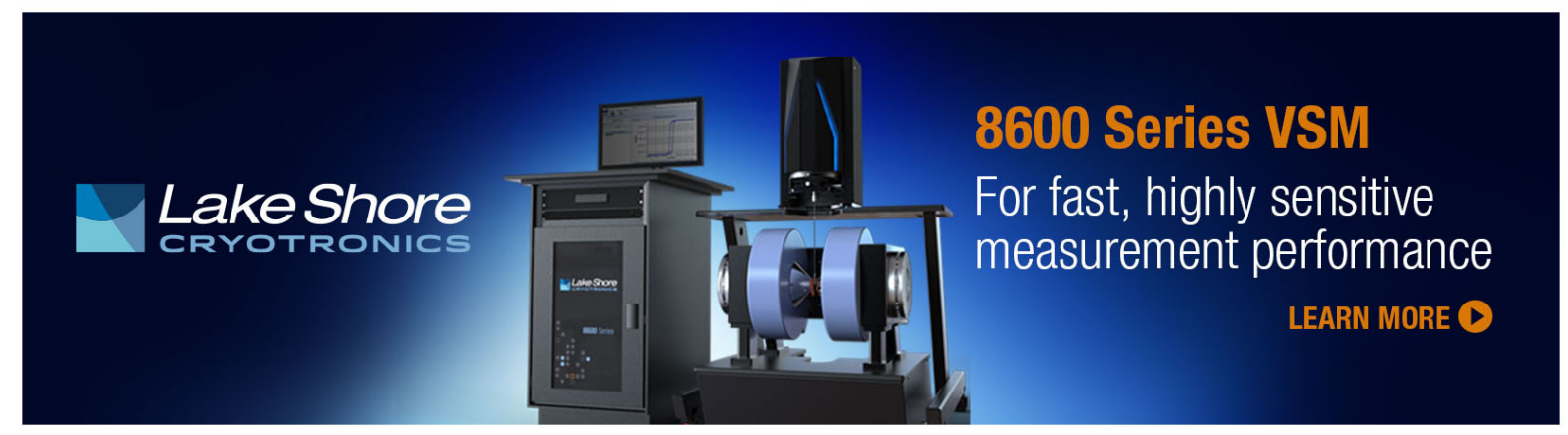




\title{
Independent tuning of excitonic emission energy and decay time in single semiconductor quantum dots
}

\author{
B. Höfer, ${ }^{1}$ J. Zhang, ${ }^{1}$ J. Wildmann, ${ }^{2}$ E. Zallo, ${ }^{1, a)}$ R. Trotta, ${ }^{2, b)}$ F. Ding,,${ }^{1,3, b)}$ A. Rastelli, ${ }^{2}$ \\ and O. G. Schmidt ${ }^{1,4}$ \\ ${ }^{1}$ Institute for Integrative Nanoscience, IFW Dresden, 01069 Dresden, Germany \\ ${ }^{2}$ Institute of Semiconductor and Solid State Physics, Johannes Kepler University Linz, Altenbergerstrasse 69 , \\ 4040 Linz, Austria \\ ${ }^{3}$ Institut für Festkörperphysik, Leibniz Universität Hannover, Appelstrasse 2, 30167 Hannover, Germany \\ ${ }^{4}$ Material Systems for Nanoelectronics, TU Chemnitz, 09107 Chemnitz, Germany
}

(Received 2 March 2017; accepted 14 March 2017; published online 10 April 2017)

\begin{abstract}
Independent tuning of emission energy and decay time of neutral excitons confined in single selfassembled $\mathrm{In}(\mathrm{Ga}) \mathrm{As} / \mathrm{GaAs}$ quantum dots is achieved by simultaneously employing vertical electric fields and lateral biaxial strain fields. By locking the emission energy via a closed-loop feedback on the piezoelectric actuator used to control the strain in the quantum dot, we continuously decrease the decay time of an exciton from 1.4 to $0.7 \mathrm{~ns}$. Both perturbations are fully electrically controlled and their combination offers a promising route to engineer the indistinguishability of photons emitted from spatially separated single photon sources. Published by AIP Publishing.
\end{abstract}

[http://dx.doi.org/10.1063/1.4979481]

Creating highly indistinguishable single photons represents one of the key challenges to implement quantum photonic technologies. Self-assembled semiconductor quantum dots (QDs) are promising candidates for such single photon sources, ${ }^{1}$ as they offer intriguing features like high indistinguishability, high brightness, ${ }^{2,3}$ and feasibility of both optical and electrical excitation ${ }^{4,5}$ For the quantum interference of single photons emitted from different QDs, the spatial and spectral overlap of the photons' wave-packets should be maximized. ${ }^{6,7}$ While the former requirement can be easily fulfilled, the latter, which includes matching of emission energies/polarizations and temporal overlap of photon wave packets, is much more demanding. This is mainly caused by small deviations in shape, size, intrinsic strain, and composition of as-grown QDs, which spoils any useful spectral overlap of their emitted photons. ${ }^{8}$ Therefore, accurate postgrowth tuning techniques are required and many different approaches have been demonstrated over the last few years.

To match the emission properties of different QDs, temperature tuning, electric-field-induced quantum-confined Stark effect (QCSE), ${ }^{7,9,10}$ laser processing, ${ }^{11,12}$ or magneticfield-induced diamagnetic/Zeeman shifts ${ }^{13,14}$ can be used. Strain fields induced by piezoelectric materials ${ }^{6,15-19}$ have also been exploited as a powerful tuning knob. The combination of different external fields — such as strain and electric fields-does not only allow the tuning bandwidth to be substantially extended, ${ }^{20}$ it also gives the possibility of controlling independently two QD parameters, such as the emission energy of exciton and biexciton confined in a QD. ${ }^{21}$ Although the spectral overlap between different QDs can be reliably achieved by employing these post-growth tuning techniques, simultaneous control of the temporal width of a

\footnotetext{
${ }^{a)}$ Present address: Paul-Drude-Institut für Festkörperelektronik, Hausvogteiplatz 5-7, 10117 Berlin, Germany.

${ }^{b)}$ Authors to whom correspondence should be addressed. Electronic addresses: rinaldo.trotta@jku.at and f.ding@ifw-dresden.de
}

photon wave-packet at constant energy is still challenging. No concepts have been proposed to ensure the temporal overlap of single photons from different QDs apart from temporal filtering. The temporal extension of the photon wave packet is given by the coherence time $\tau_{\mathrm{c}}$, which is related to the radiative decay time $\tau_{\mathrm{r}}$ and dephasing time $\tau_{\text {deph }}$ by $1 / \tau_{\mathrm{c}}=1 /\left(2 \tau_{\mathrm{r}}\right)+1 / \tau_{\text {deph. }}{ }^{22}$ In the Fourier-transform limited region, the dephasing process is minimized (for example, via the resonant excitations ${ }^{23}$ or the application of vertical electrical fields ${ }^{2,24}$ ) and therefore manipulating the decay time $\tau_{\mathrm{r}}$ is the key to improve the temporal overlap of the wave packets, and hence the photon indistinguishability. The most common technique for controlling $\tau_{\mathrm{r}}$ is the exploitation of the Purcell effect in optical microcavities, which has been shown to improve the indistinguishability of photons emitted both by the same $\mathrm{QD}^{22}$ and by independent QDs. ${ }^{25}$ However, since the lifetime of excitons confined in different as-grown QDs is different and the Purcell effect critically depends on the cavity design and position of the $\mathrm{QD}$, it is difficult to achieve a smooth tuning of $\tau_{\mathrm{r}}$ while keeping the emission energy fixed. ${ }^{26}$ Some other techniques, such as electric fields and thermal annealing, ${ }^{27}$ can be also used to manipulate $\tau_{\mathrm{r}}$, but they also lead to an unavoidable modification of the excitonic emission energy. It would be thus highly desirable to vary the decay time while fixing the emission energy at precisely defined values.

In this work, we show that the simultaneous application of electric and biaxial stress fields allows for independent tuning of emission energy and decay time in single selfassembled $\mathrm{In}(\mathrm{Ga}) \mathrm{As} / \mathrm{GaAs}$ QDs. Using an automated feedback control algorithm, we demonstrate the locking of emission energy while modulating the decay time of the neutral exciton in a single QD. The device studied in this work, which is capable of providing large vertical electric fields and lateral biaxial strain fields to the QDs, is schematically shown in Fig. 1. The sample was grown by solid source molecular beam epitaxy on a semi-insulating GaAs (001) 
(a)

(b)

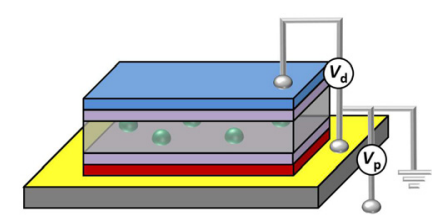

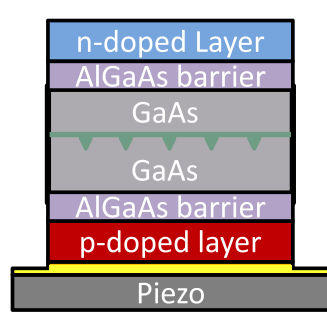

FIG. 1. Device structure for the simultaneous application of vertical electric fields and in-plane stress fields to InGaAs QDs. (a) Sketch of the device and (b) layer structure of the QD nanomembrane.

substrate. After de-oxidation and buffer layer growth, a $100 \mathrm{~nm} \mathrm{Al} 0_{0.75} \mathrm{Ga}_{0.25} \mathrm{As}$ sacrificial layer was grown for the subsequent nanomembrane release. A layer of self-assembled InGaAs QDs was deposited at the center of a $10 \mathrm{~nm} \mathrm{GaAs}$ quantum well which was cladded on both sides with $70 \mathrm{~nm}$ thick intrinsic $\mathrm{Al}_{0.4} \mathrm{Ga}_{0.6} \mathrm{As}$ layers. These layers act as barriers, which reduce carrier ionization at high electric fields across the $n-i-p$ diode structure and allow for a shift of the emission energy over a broad spectral range. ${ }^{21,28}$ The $n-i-p$ diode nanomembrane was then released from the wafer and integrated onto a PMN-PT $\left(\left[\mathrm{Pb}\left(\mathrm{Mg}_{1 / 3} \mathrm{Nb}_{2 / 3}\right) \mathrm{O}_{3}\right]_{0.72}-\left[\mathrm{PbTiO}_{3}\right]_{0.28}\right)$ piezoelectric actuator via a flip-chip transfer procedure. ${ }^{18}$

Controlled application of in-plane biaxial compressive/ tensile strain fields to the QD-containing nanomembrane can be realized by changing the voltages $V_{\mathrm{P}}$ in the range of $-200 \mathrm{~V}<V_{\mathrm{p}}<800 \mathrm{~V}$ across the $300-\mu \mathrm{m}$-thick PMN-PT substrate. A strain tuning of about $11 \mathrm{meV}$ is achieved for the studied device. In addition, by exploiting the quantum-confined-Stark-effect (QCSE), the $n-i-p$ diode nanomembrane represents an electric-field-tunable single-photon source. The design of the $n-i-p$ diode follows that in the work by Bennett et al., ${ }^{28}$ and it was shown that the main effect of small

\section{$V_{d}=0 \mathrm{~V}$ in (a) and (b)}

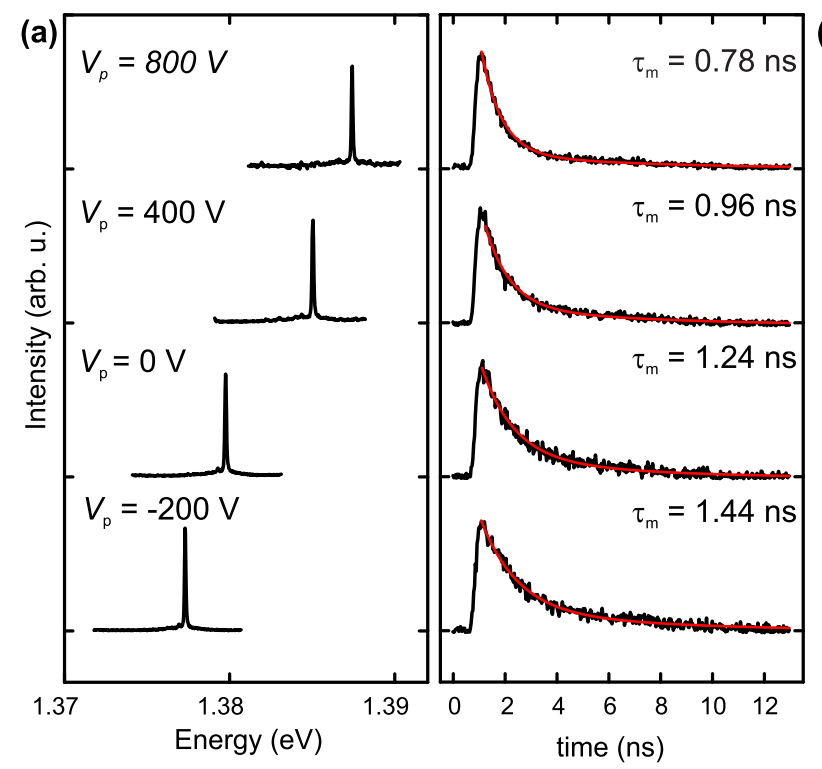

FIG. 2. Emission spectra and decay curves at different piezo voltages: (a) The neutral exciton emission $(\mathrm{X})$ shows a blue shift when the voltage on the piezo $(V p)$ is changed from $-200 \mathrm{~V}$ to $800 \mathrm{~V}$ (the diode voltage $V_{\mathrm{d}}$ is kept fixed at $0 \mathrm{~V}$ ). Note that the PL intensity fluctuations were mainly due to the strain-tuning induced defocus. (b) Time dependent spectra of the exciton line shown in (a). Red lines represent single exponential fits. vertical electric fields (in the radiative decay dominant region) on the measured decay time $\tau_{\mathrm{m}}$ is the changing exciton oscillator strength, which influences the radiative decay time $\tau_{\mathrm{r}}$ (where $1 / \tau_{\mathrm{m}}=1 / \tau_{\mathrm{r}}+1 / \tau_{\mathrm{nr}}$, and $\tau_{\mathrm{nr}}$ is the non-radiative decay time). ${ }^{9}$ At larger electric fields, a drop in $\tau_{\mathrm{m}}$ can occur, as $\tau_{\mathrm{nr}}$ decreases due to the tunneling of electrons out of the QDs.

For optical measurements, the QDs were excited at $850 \mathrm{~nm}$ directly in the wetting layer with $\sim 100$ fs pulses from a modelocked Ti:Sa laser. The micro-photoluminescence ( $\mu$-PL) spectra were recorded with a liquid-nitrogen-cooled $\mathrm{CCD}$ camera coupled to a spectrometer. A grating with 1800 lines/mm was used, with a spectral resolution of about $20 \mu \mathrm{eV}$. Time-resolved PL measurements were performed by a time correlated single photon counting module (TCSPC) together with an avalanche photodiode (APD). All measurements were carried out at $5 \mathrm{~K}$.

Using the device shown in Fig. 1, we combine the two post-growth tuning techniques to shift the emission energy of the neutral exciton $(\mathrm{X})$ in a single QD and to control independently the decay time, which is measured by time resolved spectroscopy. The excitation power was kept constant and low enough to avoid pumping of the biexciton states. While the effects of the QCSE on the exciton dynamics have been extensively investigated in previous works, there is no detailed study on the effects of in-plane strain fields on the exciton decay. Figure 2(a) shows PL spectra recorded for $V_{\mathrm{d}}=0 \mathrm{~V}$, while $V_{\mathrm{p}}$ is varied from $-200 \mathrm{~V}$ to $800 \mathrm{~V}$. For increasing piezo voltages in this range, the $\mathrm{X}$
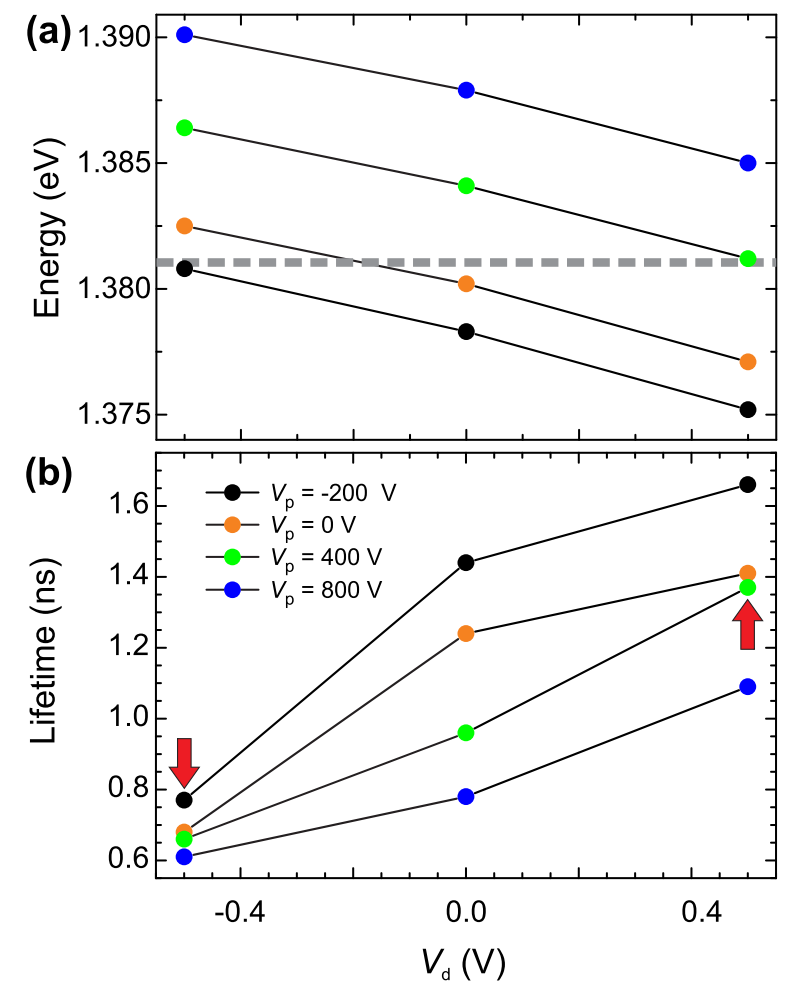

FIG. 3. X emission energy (a) and lifetime (b) for different $V_{\mathrm{p}}$ at different $V_{\mathrm{d}}$ from $-0.5 \mathrm{~V}$ to $0.5 \mathrm{~V}$. The two data points (crossing the dashed line in (a) and indicated by red arrows in (b)) represent two experimental conditions, where the emission energies are almost identical but the lifetimes are 0.77 and $1.37 \mathrm{~ns}$, respectively. In this range of $V_{\mathrm{d}}$, the radiative recombination still dominates the decay process, and the drop in decay time $\tau_{\mathrm{m}}$ is mostly due to the reduced electron and hole overlap induced by the quantum confined Stark effect. 
emission energy blue shifts from $1.377 \mathrm{eV}$ to $1.388 \mathrm{eV}$. Note that, due to a slight mechanical drift of the sample induced by the strain tuning (especially, at large voltages on the piezo), there are fluctuations in the PL intensities shown in Fig. 2(a). Time-resolved PL spectra under different strain conditions are shown in Fig. 2(b). The measured PL decay time $\tau_{\mathrm{m}}$ was determined by single exponential fitting as indicated by the red lines in Fig. 2(b). It turns out that $\tau_{\mathrm{m}}$, which sets a lower limit on $\tau_{\mathrm{r}}$, is almost halved (from $1.44 \mathrm{~ns}$ to $0.78 \mathrm{~ns}$ ) for increasing compressive strains over the entire strain tuning range. Considering that $\tau_{\mathrm{r}}$ is inversely proportional to the oscillator strength and therefore the square of the overlap of the electron and hole, ${ }^{9,29}$ the decrease in $\tau_{\mathrm{m}}$ implies an increase in the electron-hole overlap of up to $35 \%$ under biaxial compressive strain fields. In fact, we expect the externally induced compressive strain (estimated to vary by about $0.15 \%{ }^{15,16,18-21}$ ) to have negligible effects on $\tau_{\mathrm{nr}}$, since it is an order of magnitude less than the built-in strain in our QDs. An extensive theoretical simulation (to be published elsewhere) indicates that the electron lies above the hole in the studied QDs and that the piezo-induced compressive strain leads to an increased wave function overlap. This fact is mostly due to strain-induced changes in the conduction-bandoffsets, with a consequent increase of the electron confinement under compressive strain. ${ }^{15}$ Unlike the vertical electrical fields, ${ }^{2,24}$ the external strain fields do not change the charge environment. Therefore, we do not expect any significant increase of dephasing processes induced by strain.

The main results of this work are shown in Fig. 3, where emission energy (Fig. 3(a)) and decay time (Fig. 3(b)) of the neutral exciton are plotted for various values of $V_{\mathrm{p}}$ and $V_{\mathrm{d}}$. For a given $V_{\mathrm{p}}$ (i.e., fixed strain condition), the emission energy shifts by about $6 \mathrm{meV}$ when $V_{\mathrm{d}}$ is changed from -0.5 to $0.5 \mathrm{~V}$. In the meanwhile, the decay time $\tau_{\mathrm{m}}$ roughly doubles. Considering the fact that the $n-i-p$ diode design is very similar to that in the work by Bennett et al. ${ }^{28}$ we attribute the changes of $\tau_{\mathrm{m}}$ to the QCSE in a voltage range where the radiative decay still dominates. Most interestingly, the data highlighted by the red arrows and the dashed line $\left(V_{p}=-200 \mathrm{~V}\right.$ and $V_{\mathrm{d}}=-0.5 \mathrm{~V}$ vs. $V_{\mathrm{p}}=400 \mathrm{~V}$ and $V_{\mathrm{d}}=0.5 \mathrm{~V}$ ) show that the combination of the two fields allows the lifetime to be modified (from $0.77 \mathrm{~ns}$ to $1.37 \mathrm{~ns}$ ) at a fixed energy of the exciton

(a)

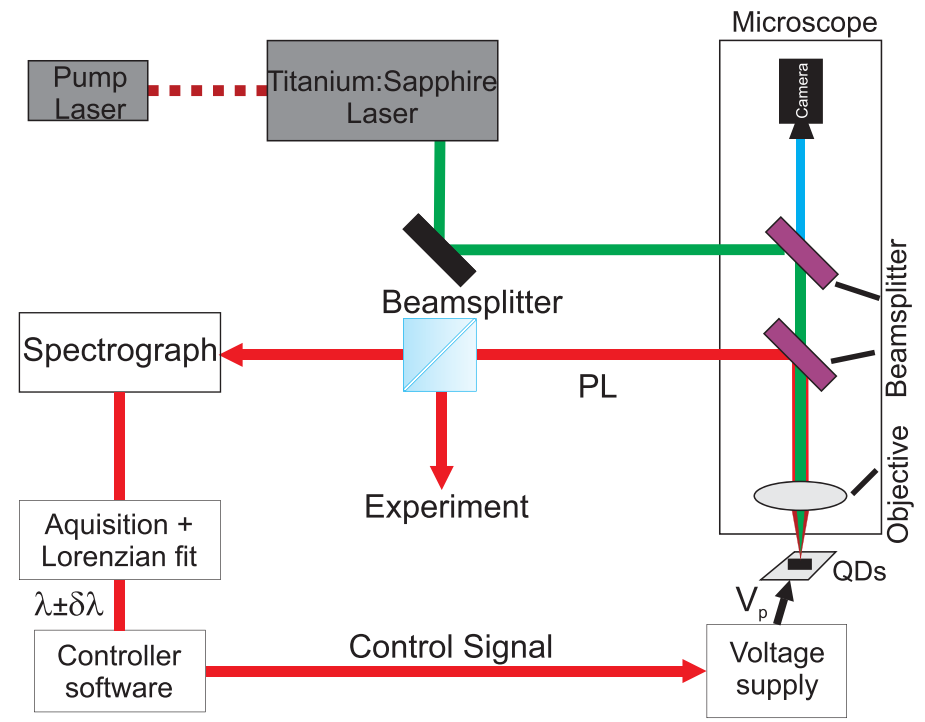

(b)

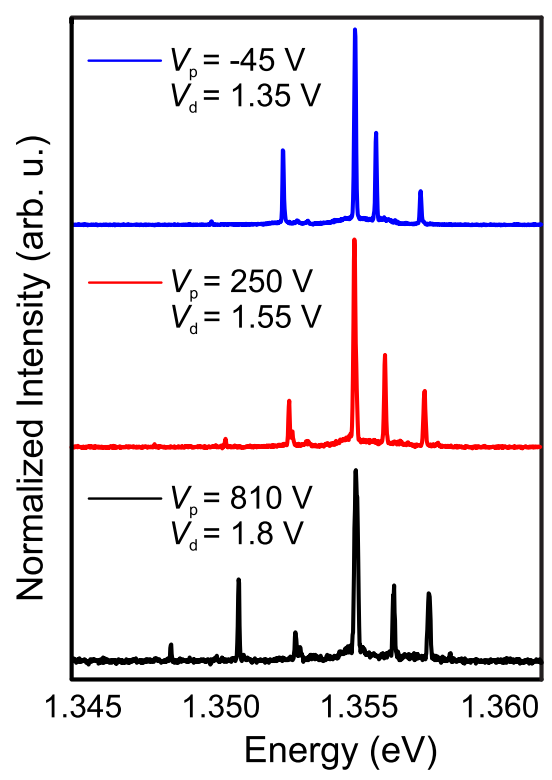

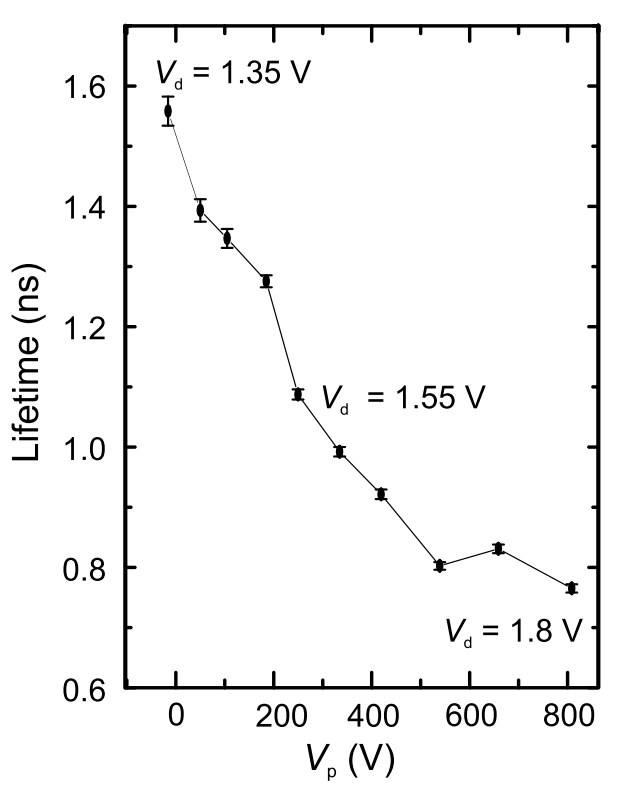

FIG. 4. (a) Sketch of the setup for the feedback-controlled PL stabilization experiment. (b) Three emission spectra of the investigated QD collected by using feedback-loop on $V_{\mathrm{p}}$, while $V_{\mathrm{d}}$ is varied. The brightest excitonic emission is kept at a constant energy during the entire experiment. (c) The lifetime of the $\mathrm{X}$ vs. different combinations of $V_{\mathrm{d}}$ and $V_{\mathrm{p}}$ during the stabilization experiment. The error bars shown in this figure are from the single exponential curve fitting procedure. 
transition (at around $1.381 \mathrm{eV}$ ). In other words, the combination of the two fields allows for independent control of the exciton lifetime and emission energy.

Due to the fact that the strain field and the electrical field influence the exciton emission energy (and the radiative lifetime) with different magnitudes, it is always possible to tune the exciton radiative lifetime at one fixed emission energy by using the method provided here. To provide a complete picture, we also biased the device at large voltages. In this experiment (performed using the configuration sketched in Fig. 4(a)), the X emission energy was monitored and actively stabilized at a constant value of $1.355 \mathrm{eV}$, similarly to Trotta et al. ${ }^{21}$ To keep the emission energy at this constant value, part of the emitted light from the QD is sent through a spectrometer and detected with a CCD camera. Fitting the spectrum with a Lorenzian curve gives the peak position of $\mathrm{X}$. After comparison with our fixed value $(1.355 \mathrm{eV})$, the peak position will be corrected with the applied voltage on the piezo. ${ }^{16}$ Figure 4(b) shows the emission spectra of the measured QD for $V_{\mathrm{d}}=1.35 \mathrm{~V}$ (blue), $1.55 \mathrm{~V}$ (red), and $1.8 \mathrm{~V}$ (black), with adapted piezo voltages to match the energies of the neutral exciton. While the exciton transition is frequency-locked at the predefined energy, other emission lines, corresponding to different charge configurations in the QD, are observed to shift due to the effects produced by strain and electric fields on the spatial distribution and interaction of electrons and holes. ${ }^{21}$ The corresponding tuning behavior of the neutral excitonic lifetime $\tau_{\mathrm{m}}$ is provided in Fig. 4(c), where we observe a very large tuning range from 0.75 to $1.55 \mathrm{~ns}$. It is worth noting that the applied $V_{\mathrm{d}}$ is larger than that in Fig. 3, and therefore, the drop in excitonic lifetime is due to the tunneling of electrons out of QDs. ${ }^{28}$ The opening of the non-radiative decay channel obviously decreases the internal quantum efficiency of QDs. Therefore, for practical applications, the device should be operated in the region where the radiative decay process is dominant.

In conclusion, we have demonstrated the independent control of the excitonic lifetime and emission energy of single InGaAs QDs embedded in an n-i-p diode structure integrated onto a piezoelectric substrate. The independent control was obtained by exploiting two complementary post-growth tuning techniques, the effect of the QCSE across the diode and biaxial strain provided by the piezoelectric substrate. We have analyzed the effects of these two perturbations and used their combination to achieve a large range of the excitonic lifetime while keeping the emission energy at a fixed value. As shown recently, the application of an electric field can be used to strongly reduce the charge noise. ${ }^{2,24}$ In this context, the method provided here can be used, to stabilize the energy of exciton emission at that of a laser for resonant pumping and to study how the indistinguishability of emitted photons can be engineered by the simultaneous applications of two perturbation fields.

The work was financially supported by the BMBF QuaHL-Rep (Contract Nos. 01BQ1032 and 01BQ1034) and Q.Com-H (16KIS0106), ERC starting grant "QD-NOMS" with No. 715770, as well as the European Union Seventh Framework Program 209 (FP7/2007-2013) under Grant Agreement No. 601126210 (HANAS). The authors thank B. Eichler, R. Engelhard, and S. Harazim for the technical support on the device fabrication.
${ }^{1}$ A. J. Shields, Nat. Photonics 1, 215-223 (2007).

${ }^{2}$ N. Somaschi, V. Giesz, L. De Santis, J. C. Loredo, M. P. Almeida, G. Hornecker, S. L. Portalupi, T. Grange, C. Anton, J. Demory, C. Gomez, I. Sagnes, N. D. Lanzillotti-Kimura, A. Lemaitre, A. Auffeves, A. G. White, L. Lanco, and P. Senellart, Nat. Photonics 10, 340-345 (2016).

${ }^{3}$ X. Ding, Y. He, Z. C. Duan, N. Gregersen, M. C. Chen, S. Unsleber, S. Maier, C. Schneider, M. Kamp, S. Hofling, C. Y. Lu, and J. W. Pan, Phys. Rev. Lett. 116, 020401 (2016).

${ }^{4}$ Y. M. He, Y. He, Y. J. Wei, D. Wu, M. Atature, C. Schneider, S. Hofling, M. Kamp, C. Y. Lu, and J. W. Pan, Nat. Nanotechnol. 8, 213-217 (2013).

${ }^{5}$ R. M. Stevenson, C. L. Salter, J. Nilsson, A. J. Bennett, M. B. Ward, I. Farrer, D. A. Ritchie, and A. J. Shields, Phys. Rev. Lett. 108, 040503 (2012).

${ }^{6}$ E. B. Flagg, A. Muller, S. V. Polyakov, A. Ling, A. Migdall, and G. S. Solomon, Phys. Rev. Lett. 104, 137401 (2010).

${ }^{7}$ R. B. Patel, A. J. Bennett, I. Farrer, C. A. Nicoll, D. A. Ritchie, and A. J. Shields, Nat. Photonics 4, 632-635 (2010).

${ }^{8}$ O. G. Schmidt, Lateral Alignment of Epitaxial Quantum Dots (Springer, Berlin, 2007).

${ }^{9}$ A. J. Bennett, M. A. Pooley, R. M. Stevenson, M. B. Ward, R. B. Patel, A. B. de la Giroday, N. Skold, I. Farrer, C. A. Nicoll, D. A. Ritchie, and A. J. Shields, Nat. Phys. 6, 947-950 (2010).

${ }^{10}$ B. D. Gerardot, S. Seidl, P. A. Dalgarno, R. J. Warburton, D. Granados, J. M. Garcia, K. Kowalik, O. Krebs, K. Karrai, A. Badolato, and P. M. Petroff, Appl. Phys. Lett. 90, 041101 (2007).

${ }^{11}$ M. Benyoucef, L. Wang, A. Rastelli, and O. G. Schmidt, Appl. Phys. Lett. 95, 261908 (2010).

${ }^{12}$ A. Rastelli, A. Ulhaq, S. Kiravittaya, L. Wang, A. Zrenner, and O. G. Schmidt, Appl. Phys. Lett. 90, 073120 (2007).

${ }^{13}$ A. J. Hudson, R. M. Stevenson, A. J. Bennett, R. J. Young, C. A. Nicoll, P. Atkinson, K. Cooper, D. A. Ritchie, and A. J. Shields, Phys. Rev. Lett. 99, 266802 (2007).

${ }^{14}$ R. M. Stevenson, R. J. Young, P. See, D. G. Gevaux, K. Cooper, P. Atkinson, I. Farrer, D. A. Ritchie, and A. J. Shields, Phys. Rev. B 73, 033306 (2006).

${ }^{15}$ F. Ding, R. Singh, J. D. Plumhof, T. Zander, V. Krapek, Y. H. Chen, M. Benyoucef, V. Zwiller, K. Dorr, G. Bester, A. Rastelli, and O. G. Schmidt, Phys. Rev. Lett. 104, 067405 (2010).

${ }^{16}$ A. Rastelli, F. Ding, J. D. Plumhof, S. Kumar, R. Trotta, C. Deneke, A. Malachias, P. Atkinson, E. Zallo, T. Zander, A. Herklotz, R. Singh, V. Krapek, J. R. Schroter, S. Kiravittaya, M. Benyoucef, R. Hafenbrak, K. D. Jons, D. J. Thurmer, D. Grimm, G. Bester, K. Dorr, P. Michler, and O. G. Schmidt, Phys. Status Solidi B 249, 687-696 (2012).

${ }^{17}$ S. Seidl, M. Kroner, A. Hogele, K. Karrai, R. J. Warburton, A. Badolato, and P. M. Petroff, Appl. Phys. Lett. 88, 203113 (2006).

${ }^{18}$ R. Trotta, P. Atkinson, J. D. Plumhof, E. Zallo, R. O. Rezaev, S. Kumar, S. Baunack, J. R. Schroter, A. Rastelli, and O. G. Schmidt, Adv. Mater. 24, 2668-2672 (2012).

${ }^{19}$ E. Zallo, R. Trotta, V. Krapek, Y. H. Huo, P. Atkinson, F. Ding, T. Sikola, A. Rastelli, and O. G. Schmidt, Phys. Rev. B 89, 241303(R) (2014).

${ }^{20}$ R. Trotta, E. Zallo, C. Ortix, P. Atkinson, J. D. Plumhof, J. van den Brink, A. Rastelli, and O. G. Schmidt, Phys. Rev. Lett. 109, 147401 (2012).

${ }^{21}$ R. Trotta, E. Zallo, E. Magerl, O. G. Schmidt, and A. Rastelli, Phys. Rev. B 88, 155312 (2013).

${ }^{22}$ S. Unsleber, D. P. S. McCutcheon, M. Dambach, M. Lermer, N. Gregersen, S. Hofling, J. Mork, C. Schneider, and M. Kamp, Phys. Rev. B 91, 075413 (2015).

${ }^{23}$ A. Muller, E. B. Flagg, P. Bianucci, X. Y. Wang, D. G. Deppe, W. Ma, J. Zhang, G. J. Salamo, M. Xiao, and C. K. Shih, Phys. Rev. Lett. 99, 187402 (2007).

${ }^{24}$ A. V. Kuhlmann, J. H. Prechtel, J. Houel, A. Ludwig, D. Reuter, A. D. Wieck, and R. J. Warburton, Nat. Commun. 6, 8204 (2015).

${ }^{25}$ V. Giesz, S. L. Portalupi, T. Grange, C. Anton, L. De Santis, J. Demory, N. Somaschi, I. Sagnes, A. Lemaitre, L. Lanco, A. Auffeves, and P. Senellart, Phys. Rev. B 92, 161302(R) (2015).

${ }^{26}$ C. Y. Jin, R. Johne, M. Y. Swinkels, T. B. Hoang, L. Midolo, P. J. van Veldhoven, and A. Fiore, Nat. Nanotechnol. 9, 886-890 (2014).

${ }^{27}$ W. Langbein, P. Borri, U. Woggon, V. Stavarache, D. Reuter, and A. D. Wieck, Phys. Rev. B 70, 033301 (2004).

${ }^{28}$ A. J. Bennett, R. B. Patel, J. Skiba-Szymanska, C. A. Nicoll, I. Farrer, D. A. Ritchie, and A. J. Shields, Appl. Phys. Lett. 97, 031104 (2010).

${ }^{29}$ B. Alen, J. Bosch, D. Granados, J. Martinez-Pastor, J. M. Garcia, and L. Gonzalez, Phys. Rev. B 75, 045319 (2007). 